\title{
Employing Podcasts for Augmented Learning Using Information Centric Networks
}

\author{
Alina Hang ${ }^{1}$, Fernando Almeida ${ }^{2}$, Helder Castro ${ }^{2}$, Maria T. Andrade ${ }^{2}$, Leonardo \\ Chiariglione $^{3}$, Nicola Blefari-Melazzi ${ }^{4}$, Heinrich Hussmann ${ }^{1}$ \\ ${ }^{1}$ University of Munich, Media Informatics Group, Munich, Germany \\ ${ }^{2}$ INESC TEC, Porto, Portugal \\ ${ }^{3}$ CEDEO, Turin, Italy \\ ${ }^{4}$ University of Rome "Tor Vergata”, Department of Electronic Engineering, Rome, Italy
}

\begin{abstract}
With the advances in ICT (information and communication technology) online podcast have gained popularity in recent years. Usually podcasts consist of audio or video recordings which sometimes are accompanied by additional content, e.g. presentation slides. A new approach is to embed these podcasts within a social learning environment where users discuss and share annotations with each other. There are already a variety of free and proprietary solutions on the market. However, almost all solutions have different underlying technologies which make it difficult to connect those applications for collaboration (e.g. sharing of content across applications). In this paper we show how information centric networks can be used to support and facilitate the collaboration between applications and how the described limitations can be addressed. We also present the results of a first user study and report the perception of participants about this new approach.
\end{abstract}

\section{Introduction}

With the appearance of the Internet it became possible to create virtual learning environments supported through models of bi-directional communication (synchronous and asynchronous). This has enabled the development and the exponential increase of online courses. Information and communication technology (ICT), has thus created new possibilities for sharing and building knowledge.

The virtual learning environment, traditionally organized around learning platforms, began to make room for new resources and free services, which were available on the Web and could be accessed without any substantial computer knowledge. In fact, teachers and students involved in e-learning courses, can today rely on a series of tools from the new Internet generation called Web 2.0.

The term 'Web 2.0' was officially coined in 2004 by Dale Dougherty, during a team discussion on a potential future conference about the web [1]. It refers to a second generation of the web, where it is conceptualized as a technology for interactive communication. Web 2.0 has brought a dramatic change in the way we use the Internet, as it offers us several new tools and services that allow easier interaction and participation of all users. O'Reilly [1], thus, observes that the operational progresses in the Web have evolved personal web-pages into blogs, encyclopedia into Wikipedia, text-based tutorials into streaming media applications, taxonomies into folksonomies, and questionanswer/email customer support into instant messaging services. The implications of this Web usage, and operational, revolution are enormous.

Web 2.0 covers a wide range of technologies, and many forms of Internet applications currently embody Web 2.0 qualities. The most widely used tools are blogs, wikis, podcasts, information tagging, prediction markets, and social networks [2]. A short analysis of the potential of these technologies is presented in table 1.

TABLE I. WEB 2.0 TECHNOLOGIES [3]

\begin{tabular}{|l|l|l|}
\hline \multicolumn{1}{|c|}{$\begin{array}{c}\text { Web 2.0 } \\
\text { technologies }\end{array}$} & \multicolumn{1}{|c|}{ Description } & \multicolumn{1}{c|}{$\begin{array}{c}\text { Category of } \\
\text { technology }\end{array}$} \\
\hline $\begin{array}{l}\text { Wikis, shared } \\
\text { workspaces }\end{array}$ & $\begin{array}{l}\text { Facilitates co- } \\
\text { creation of } \\
\text { contents across } \\
\text { large and } \\
\text { distributed set of } \\
\text { participants. }\end{array}$ & $\begin{array}{l}\text { Broad } \\
\text { collaboration. }\end{array}$ \\
\hline $\begin{array}{l}\text { Blogs, podcasts, } \\
\text { videocasts }\end{array}$ & $\begin{array}{l}\text { Offers individuals } \\
\text { a way to } \\
\text { communicate and } \\
\text { share information } \\
\text { with other people. }\end{array}$ & $\begin{array}{l}\text { Broad } \\
\text { communication. }\end{array}$ \\
\hline $\begin{array}{l}\text { Prediction markets, } \\
\text { polling }\end{array}$ & $\begin{array}{l}\text { Harnesses the } \\
\text { power of } \\
\text { community and } \\
\text { generates a } \\
\text { collectively } \\
\text { derived answer. }\end{array}$ & $\begin{array}{l}\text { Collective } \\
\text { estimation. }\end{array}$ \\
\hline $\begin{array}{l}\text { Tagging, user } \\
\text { RSS }\end{array}$ & $\begin{array}{l}\text { Add additional } \\
\text { information to } \\
\text { primary content to }\end{array}$ & Metadata creation. \\
\hline
\end{tabular}




\begin{tabular}{|l|l|l|}
\hline & $\begin{array}{l}\text { prioritize } \\
\text { information. }\end{array}$ & \\
\hline $\begin{array}{l}\text { Social networking, } \\
\text { network mapping }\end{array}$ & $\begin{array}{l}\text { Leverages } \\
\text { connections } \\
\text { between people to } \\
\text { offer new } \\
\text { applications }\end{array}$ & Social graphing. \\
\hline
\end{tabular}

\subsection{From Web 2.0 to e-Learning 2.0}

Linh [4] observes that tools of the Web 2.0 have been strongly applied in the field of communication, entertainment and collaboration. However, many of these tools, namely blogs, wikis, tagging/bookmarking, podcasts, RSS, etc. also have strong implications in the learning practices and collaboration of today's students. For instance: teachers may employ these tools in their current teaching practices to engage students as active collaborators in their learning, and thus increase their teaching efficiency: the Blog is one of the most powerful tool for sharing ideas; Wikis are most useful to facilitate group planning and collaborative construction of knowledge; podcasts are useful for publishing audio records of interviews, speeches, classes, etc.; vodcasts add the dimension of video and/or images; while RSS feeds make it easy for teachers and students to track updates on websites, posts on blogs, collaborations on wikis, and audio/video recordings on vod/(pod)casts. The above stated examples, thus, demonstrate the potential of Web 2.0 tools for e-learning.

The term e-Learning 2.0 describes, therefore, the new generation of e-Learning that followed the change of paradigm in the Web [5]. According to Silva et al. (2008), the benefits of Web 2.0 tools and services as online learning environment, can be summarized as follows:

- Promotes better teacher-student relationships and communication in a friendly environment;

- Helps students feel more comfortable about expressing themselves without embarrassment;

- Provides tools that stimulate students' enthusiasm in writing, forming opinions, peer evaluation through debate;

- Promotes collaborative work;

- Increases active intervention, which promotes students' self-confidence.

It is argued that the explosion in the popularity of this new Web paradigm was due to ease of access to the Internet and the wide availability of cheaper broadband as well as the ease of use of the content's creation tools with free applications from the Web [6]. These applications will soon be widely available, on a variety of hardware, particularly in mobile and ubiquitous platforms. The emergence of online distance education tools and e-Learning 2.0 has also been enhanced by the easy access to databases through JavaScript, Java or Flash, the language XML and RSS.

In fact, almost all characteristics of the Web 2.0 can be, advantageously, applied to e-learning within a Learning Content Management System (LCMS) or platforms like Moodle, for the price of a "platform lock-in" regarding the system chosen. Interaction with, and among, students is more active and, because of built-in interactivity, students are offered new possibilities to become involved in, and to interact with content [7].

\subsection{The new learning paradigms}

The younger generation, also called the New Millenium Learners (NML), which grew up surrounded by digital media, displays significantly different learning styles from previous cohorts. According to Pedró [8], the NML display complex learning styles that are shaped by the ubiquity, accessibility and ease of use, of digital resources. Compared to previous generations of learners, they are digitally literate, think more visually and in a non-linear manner, practice multitasking, give preference to multimedia environments and are continuously connected with their peers [8]. In learning environments, these individuals are easily bored, need a variety of stimuli not to get distracted, are impatient and expect instant feedback and rewards [9].

Current learners live in a world that is characterized by information overload [10]. By its nature, the Web rewards the comparison of multiple sources of information, which are individually incomplete and collectively inconsistent. This induces learning based on seeking, sieving, and synthesizing, rather than on assimilating a single validated source of knowledge as from books or professor's lectures [10]. According to Bruns \& Humphreys [11] current practices are characterized by the (co-) production of content by the user assisted by many social computing applications. They argue that education has to respond to these new working styles by emphasizing certain skills and attitudes in terms of their component of creativity, collaboration, critical capacity and communication.

Table 2 puts together the new skills for learning in a knowledge-based society mentioned in several publications.

TABLE II. NEW SKILLS FOR LEARNING IN A KNOWLEDGEBASED SOCIETY [12]

\begin{tabular}{|l|l|l|}
\hline \multicolumn{1}{|c|}{ Siemens (2006) } & \multicolumn{1}{|c|}{$\begin{array}{c}\text { Bruns \& } \\
\text { Humphreys } \\
\mathbf{( 2 0 0 7 )}\end{array}$} & $\begin{array}{c}\text { European Council } \\
\mathbf{( 2 0 0 6 )}\end{array}$ \\
\hline $\begin{array}{l}\text { Managing } \\
\text { information }\end{array}$ & $\begin{array}{l}\text { Creativity and } \\
\text { collaboration }\end{array}$ & $\begin{array}{l}\text { Digital } \\
\text { competence; } \\
\text { Learning to learn }\end{array}$ \\
\hline Networking & Communication & Social and civic \\
\hline
\end{tabular}




\begin{tabular}{|l|l|l|}
\hline & & competences \\
\hline $\begin{array}{l}\text { Criticial and } \\
\text { creative skills }\end{array}$ & Critical capacity & $\begin{array}{l}\text { Sense of initiative } \\
\text { and } \\
\text { entrepreneurship }\end{array}$ \\
\hline
\end{tabular}

This paper presents the use of podcasts in an augment learning scenario based in information centric networks. Section II analyzes the podcast state of the art in terms of value chain and discusses some educational podcast implementations. Section III presents the major concepts behind the information centric paradigm. Then, section IV makes a comparison with traditional implementation of podcast platforms. Furthermore, section V presents the implementation of two educational prototypes. After that, section VI demonstrates the usefulness of our proposed solution and shows the main results obtained in our focus group. Finally, section $\mathrm{V}$ draws conclusions and presents some direction for future work.

\section{State of the Art}

\subsection{Value chain}

Podcasts follow a simple publish-and-subscribe model where there is a user responsible for publication of a digital content and other users who assume the role of subscribers or listeners. Publishers are loosely coupled to subscribers. With the topic of the digital content being the focus, publishers and subscribers are allowed to remain uninformed of system topology and of each other.

The process for podcasting and vodcasting can be broken down into five steps as it is illustrated in figure 1 .

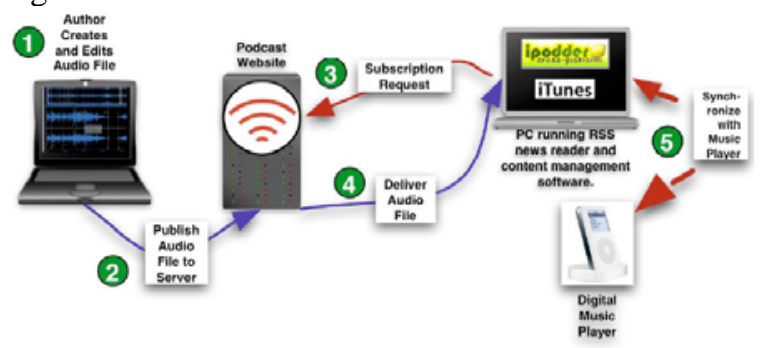

Figure 1. Podcasting publishing and subscribe model [13]

The complexity of those steps is dependent on the purpose of the content and the quality level at which it will be produced.

The initial step, in the production of a podcast, includes planning, writing, and recording content, as well as audio/video editing and file compression. Production requires recording hardware, like digital microphones and digital cameras, and software for editing audio and/or video segments. In addition to the relevant audio/video files, the creator must generate an RSS feed. A feed is a simple XML file that lists the location of podcast episodes. It also includes information about the podcast, such as publish dates, titles, and descriptions of the series and of each episode [14]. The podcast creator can write the feed manually, or use software to generate the feed file.

In a traditional approach, the audio/video file and RSS feed are then posted to a Web server. For the first podcast in a series, and for ongoing series that are always open to new subscribers, the podcast creator must notify the audience of the existence of the podcast by publicizing the location of the RSS feed. Many podcasters post a link to the RSS feed on their blogs, Web sites, or other public Web spaces. The podcast creator can also list information about the podcast in one of many directories that categorize podcasts alphabetically or by topic. The listener is able to subscribe to the podcast series using a podcast aggregator (software that checks podcast feeds for updates at specific intervals). This step is only necessary the first time. Once the listener has subscribed to the podcast, they will remain subscribed until they choose to unsubscribe.

When the listener adds a new RSS feed, the aggregator downloads all episodes referenced in the current RSS feed. At regular intervals thereafter, the aggregator checks the feed for updates and downloads any episodes added since the previous check. Listeners can access podcasts directly on their computers, or on their portable MP3/video devices. For those who prefer to listen on portable devices, most podcast aggregators will synchronize with such devices automatically. Currently, there are a significant number of software tools that are available for managing podcasts or any other audio/video content. Content Management Systems (CMS) software can be used by the community to sort and organize their content into "playlists" which can be scheduled to automatically synchronize with a media player [15].

\subsection{Podcasting implementations}

There are several podcast services available on the market. In general, one can distinguish between services that provide and distribute educational content, and services that allow the augmentation of educational content (e.g. with comments). The following sections provide an overview of existing solutions and depict their advantages and limitations.

\section{Providing Educational Content and Redistribution:}

a) iTunes $U^{1}$ is an initiative by Apple that supports lecturers in publishing educational content over the iTunes software. The service is based on a publish-subscribe model, where students subscribe to podcasts of their interest and are notified when new episodes are released.

\footnotetext{
${ }^{1}$ http://www.apple.com/de/education/itunes-u/
} 
b) MIT has a service called OpenCourseWare ${ }^{2}$ that offers a variety of creative common content for education, ranging from lecture notes to podcasts.

c) The technical university of Darmstadt has a similar service with podcasts that are published under the creative common license.

d) EL@N UK Campus ${ }^{3}$ is a service by the University of Nottingham. It provides a package of information and communication technologies to enhance teaching and learning. This platform aims to provide core material online, broaden the range of learning activities for students and encourage collaborative learning. This platform supports academics in the creation and delivery of e-Learning materials though Virtual Learning Environments (VLEs) and online assessment tools.

The described services are exemplary solutions to distribute educational content over the Internet. All of them have their advantages, but also some shortcomings. For example, in order to use the iTunes $U$ service, students are forced to utilize the iTunes platform in order to download podcasts to their local device. Connecting other applications such that they can access iTunes podcasts is difficult, on the one hand due to the different implementation technologies that are used to implement the applications, but also because of the protection of those contents through licenses. MIT and the technical university of Darmstadt provide their content under the creative common license. However, the license information cannot be directly bound within the file, but is placed on the website close to it, meaning that the license is easily lost once the podcast is downloaded and also tracking of redistribution is not directly supported (e.g. how often has the file been redistributed).

Interactive Learning Platforms:

An innovative idea to enhance traditional podcasts is to augment them with additional features inspired by the Web2.0 paradigm. Some of these solutions are presented in the following.

a) Unterrichtsmitschau ${ }^{4}$ at the university of Munich provides an online learning environment with interactive features where students collaborate with each other by annotating podcast while watching them. b) In Fall 2012 MIT will launch their product called MITx $^{5}$ which is an interactive learning platform aiming at enhancing the educational experience.

c) One of the best-known course management systems is Moodle ${ }^{6}$. It is an open source software used by many universities and allows individual modifications through the installation of plugins (e.g. the installation of podcasting modules).

d) Blackboard Learn, Blackboard Collaborate, Blackboard Mobile ${ }^{7}$ are platforms designed by Blackboard for course management, video and audio conferencing, and mobile e-Learning, respectively. Although the Blackboard software is proprietary, developers are able to extend the functionality of the system, and create customized course management and delivery by developing software and applications known as Building Blocks. Additionally, in 2011 Blackboard launched CourseSites, a free version of its Blackboard Lean and Collaborative software.

The four above platforms are only a set of examples out of many other commercial and noncommercial applications. They all have common points (management of educational content and adding interactive features). Thus, it would be desirable to connect these applications with each other (e.g. when universities and companies want to collaborate and share content with each other within their own applications). However, each of the applications uses different technologies, underlying different infrastructures, making the collaboration cumbersome. Seen more generally, all the systems in nowadays' use put the emphasis wrongly, by forcing the user to first choose a technology and then the content, whereas users are mainly interested in content. So it makes sense to discuss a paradigm change putting access to information in the center of the whole user experience. The way how so-called "information centric networks" can address these issues will be described in section 5 . In order to understand the basic ideas of an information centric network, the next section gives a brief overview of the most important aspects.

\section{The Information Centric Paradigm}

\subsection{Design issues and principles}

The Internet was originally conceived as an "Internet of Hosts", whose underlying protocols were designed to support the exchange of simple unstructured information items, between wellidentified nodes. Today, by contrast, it is becoming an "Internet of Things" (devices and appliances associated to their own IP addresses), an "Internet of

\footnotetext{
${ }^{5}$ http://mitx.mit.edu/

${ }^{6}$ http://moodle.org/

${ }^{7}$ http://www.blackboard.com/platforms/learn/overview.aspx
} 
Services" (in which users in different localities access different functionalities on different hosts), an "Internet of Media" (shared and managed across different networks) and an "Internet of People" (boosted by the explosion of social networking and the emergence of the Web 2.0 paradigm). In these "new Internets", the key elements are no longer "hosts" but data and services (or content).

This is an interesting paradigm shift that increasingly puts information dissemination, through a vast set of heterogeneous devices (e.g. mobile phones, tablets, etc.) into the center of user activities, whereas network technology and software platforms become utilities the user does not want to know about. This observation poses a new and demanding challenge in terms of establishing a well-defined content awareness structure.

Information-centric networking (ICN), also known as content-aware, content-centric or dataoriented networking, tries to deal with the lack of a global content naming and addressing scheme, as well as with the need for unified content access and consumption. At the same time, such networks can be made aware of the type of content they are transporting and, thus, are able to adapt and offer the appropriate quality of experience to the end users which is not easily possible with nowaday's contentagnostic network technologies.

The proposed benefits of content/informationcentric networking are wide. Within such a system, content would be uniquely addressed with the ability to be accessed from any location using a simple request/reply paradigm. According to Tyson [16], this would make application development simpler and allow an array of network-level optimizations to be performed relating to the storage and distribution of content. While imaginative solutions through incremental changes to the network stack or overlay networks have been successfully proposed to address these new usage patterns, it is widely admitted in literature that these solutions present, also, limitations and drawbacks, in terms of their scalability, security, mobility, and manageability, which hinder their ability to support the creation or deployment of more ambitious and innovative services [17]-[18].

\subsection{The CONVERGENCE framework}

CONVERGENCE proposes [19] to enhance the Internet with a novel, content-centric, publishsubscribe service model, based on the Versatile Digital Item (VDI), which is a common container for all kind of digital content, derived from the MPEG21 standard [20]. VDIs will serve the new needs of the "Internet of Things" providing a homogeneous way of searching and handling structured information, incorporating privacy and copyright protection mechanisms to support the needs of both information providers and information consumers.

The key features of the CONVERGENCE framework include the following:

- A new fundamental unit of distribution and transaction based on in the concept of VDI;

- New network functionality that supports the generation of VDI identifiers, publishing named VDIs to the network, subscription to named VDIs, and discovery and routing of the VDIs based on their names, independently of their physical location on the network;

- New open source middleware that implements a package of tools and applications to exploit the networking functionality described above;

- Community Dictionary Service (CDS) that provides an RDF-based mechanism supporting interpretation and search for userdefined metadata and tags;

- Use of a fractal concept that enables the semantic categorization of content. It is supported by the CDS to efficiently distribute VDIs across de network;

- Security and privacy mechanisms to enforce privacy-aware access control to the contents of VDIs, and a transparent and automated authentication of VDIs transported across the network.

The CONVERGENCE framework will significantly extend opportunities for interactions among different actors along the value chain for digital items (DIs). In a traditional media value chain the process is sequential. Actor 1 creates the DI, defining the title of the video, rights and permissions (meta-information). Actor 2, a dubbing company, adds sound tracks (additional resources). Actor 3, the censorship board, adds information on the video's certification (new meta-information). The chain continues until DI reaches the final Actor (i.e., consumer). The only way to edit or read a DI is locally, when it is physically available on a given actor's premises. A downstream actor has no way of modifying choices that have been made upstream. Once the DI has been delivered to the next actor down the chain there is no way the upstream actor can update the information he or she has provided.

In CONVERGENCE, by contrast (see figure 2), VDIs are living objects, that can always be modified by any authorized actor in the value chain. CONVERGENCE extends the life of the value chain beyond the actions performed in the first delivery phase (i.e., when the VDI physically reaches the consumer for the first time), allowing authorized authors to operate on VDIs remotely, even when the VDIs are physically located on the premises of other actors. CONVERGENCE's value chain can be viewed as a logical "bus" allowing any station attached to the bus (any actors), to interact with any other station. 


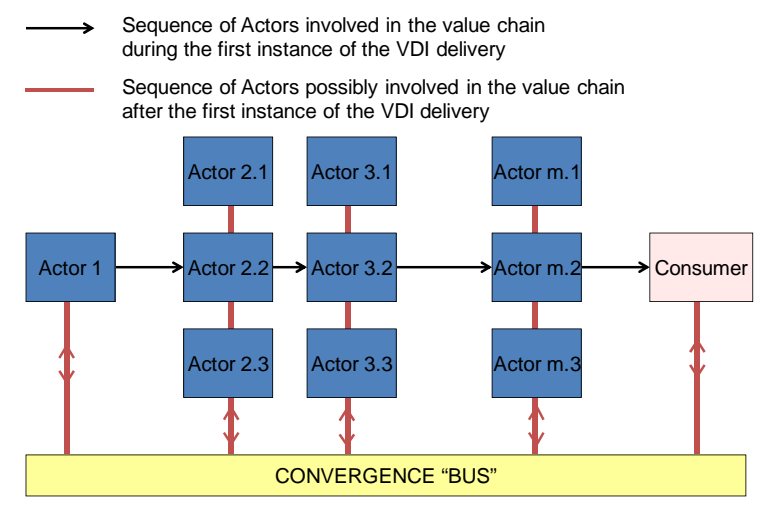

Figure 2. Sequence of actors in the CONVERGENCE value chain

\section{Comparison with Traditional Implementations}

In section 2.2 we provided an overview of podcast implementations, followed by the introduction of an information centric network called CONVERGENCE. One of the most interesting questions is how these two things can be combined and which advantages are offered to the corresponding applications? In general, CONVERGENCE does not add new functionality to the applications, but it fundamentally changes how those functionalities are achieved, opening new ways for cooperation between applications.

Each of the presented applications is based on their own technical solution. For example, some of them use Adobe Air and Flash, while others use PHP, etc. The diversity of these technical approaches to realize podcasting applications makes a connection between those applications cumbersome. Nevertheless, collaboration is desirable. Collaboration connects people from geographically different regions, it connects people with different educational background, it enhances student-tostudent communication and makes content available to a larger group. Using an information centric network like CONVERGENCE has the ability to overcome these limitations, since it provides a common infrastructure that applications can rely on. Content is stored in the CONVERGENCE network and makes it available to all applications through their representations as VDIs, still respecting the licenses that is applied to the content.

CONVERGENCE also supports the process of content creation. CONVERGENCE has a Community Dictionary Service (CDS) that helps creators in describing their content in more detail by proposing appropriate keywords from ontologies. Describing content is important, as it helps to better find content that suits the searchers needs. Another important point is the protection of intellectual property. Using CONVERGENCE, the license is strictly bound the the actual resource, respecting the rights of the creator.

Since VDIs are living objects within the CONVERGENCE frameworks, they can be easily modified by authorized users, even when the VDI is physically located on other devices. Changes are automatically communicated to users, assuring the quality of distributed content and making other notification mechanism like RSS-feeds obsolete as CONVERGENCE integrates the concepts of event reports, that shifts the functionality of notifications from the application level to the middleware level, facilitating the work for actos in the podcasting value chain. CONVERGENCE has an integrated notification mechanism that takes advantage of semantic metadata to identify the relationship between VDIs.

Altogether, CONVERGENCE provides a set of features that facilitats the creation process of podcasts. How this can be achieved within social learning environments will be described in the following Implementation section.

\section{Implementation}

In order to exploit the described features and show the differences to current implementations, we developed two educational prototypes. On the one hand, there is a podcast creator application that is used by content producers (in our case: lecturers). On the other hand, there is an augmented lecture podcast application that is used by content consumers (in our case: students). The following section describes the basic functionality of the applications and shows some examples on how to use CONVERGENCE when developing applications. The examples will show that only a few lines of codes are sufficient to achieve a powerful functionality.

\section{Podcast Creator}

The creation of podcasts is done with the help of a variety of CONVERGENCE tools (a collection of wrappers that consist of frequently used methods to communicate with the CONVERGENCE middleware). Each podcast consists of three components: video, slides, and synchronization information. Within the CONVERGENCE framework, each podcast component is represented as a VDI. It contains all the important information. Additionally, another VDI is created to put all podcast components in relation, meaning that each podcast knows where to find each of its components - the video VDI, slide VDI and synchronization VDI. In general, the publication of a VDI consists of three steps. Firstly, describing the VDI and defining the resource to be added (e.g. video file). Secondly, creating the VDI which is stored locally or on the server, but which is not yet published. Thirdly, publishing the VDI to the CONVERGENCE 
network. Figure 3 and 4 demonstrate the basic steps to accomplish the creation and publication of a VDI (in this case a podcast VDI).

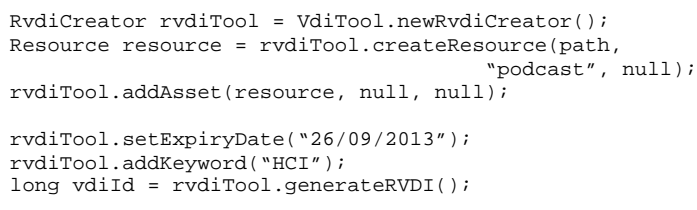

Figure 3. Exemplary Code for VDI Creation.

CONVERGENCE provides a VdiTool. It is used to instantiate the RvdiCreator which offers a set of methods that are needed in order to create a VDI. This includes embedding the resource, setting the expiration date and describing the resource using keywords. The method generateRVDI() creates the VDI and returns an ID that is needed for publication.

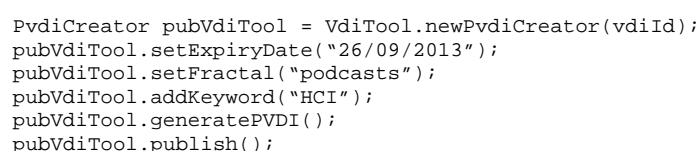

Figure 4. Exemplary Code for VDI Publication.

Publication of a VDI is achieved by creating a publication VDI, which is a special type of VDI that references to the resource VDI created in the first step. Only if there is a publication VDI, the resource VDI will be available within the CONVERGENCE network. The VdiTool has a PvdiCreator that comprises all important methods for publication. It provides methods to set the metadata within the VDI (expiration date, keywords, and fractals) and to publish the VDI. The concept of fractals is probably one of the most interesting. It can be used to categorize content semantically. Thus, only users who subscribe to the same fractals will get notifications about new publications.

The given examples are the core functions of CONVERGENCE. However, there are still many other features, e.g. the revocation or update of VDIs. Deleting or Updating content from the CONVERGENCE network reassures that users who have downloaded the podcasts are notified about changes in case the VDI has been updated.

\section{Augmented Lecture Podcasts}

Complementary to the podcast creator, we also developed an augmented lecture podcast service. As described before, augmented lecture podcasts are based on traditional podcast, but are extended with interactive features. For this application, we embedded lecture podcasts into a social learning environment where students add annotations of private, semi-private and public nature while watching podcasts. Figure 5. shows a screenshot of the application.

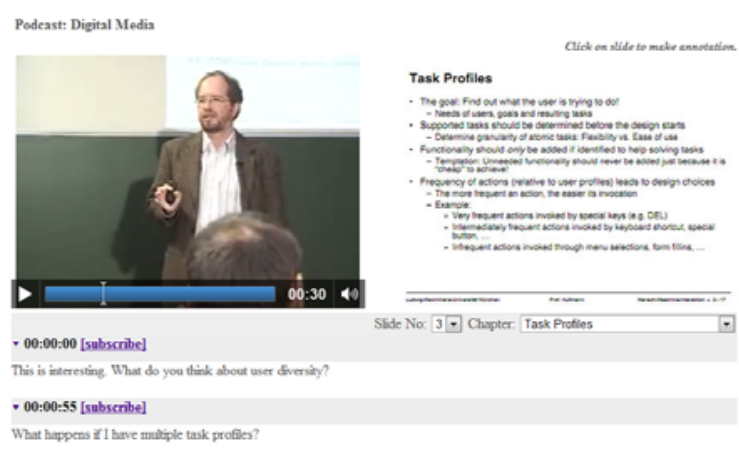

Figure 5. Screenshot of ALP application. Video (left) with synchronized slides (right) and annotations by students (bottom).

The service uses all podcasts that are published by the podcast creator application. In order for students to find a podcast, they have to perform a search. In general, searching for podcasts is nothing else than a subscription. Therefore users have to enter keywords and submit their search. This will initiate the process of creating a subscription VDI. It is also a special type of VDI that contains the keywords for searching. These VDIs are used to be matched with publication VDIs. When the VDIs are in the same fractal and have the same keywords, a match has been found and will be displayed to the user. The advantage of this approach is that it also supports the asynchronous search. This means, that users are notified when new publications are done after their search. Figure 6 shows exemplary code to create a subscription VDI.

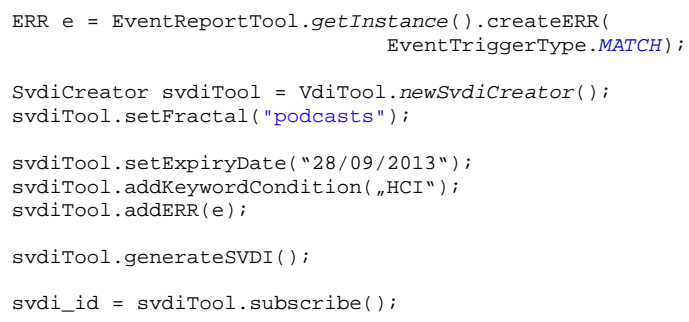

Figure 6. Exemplary Code for VDI Subscription.

Again, the VdiTool is used, this time to instantiate the SvdiCreator. The provided methods allow the selection of fractals, the definition of keywords and expiration date. In comparison to the creation of publication VDIs, subscription VDIs are based on so-called event reports. Thus, the EventReportTool is needed to create an event report and to include it into the subscription VDI. The event report is the core mechanism to notify users once a subscription matches with a publication. 


\section{User Study}

In the previous section, we presented two applications - the podcast creator and the augmented podcast service. We also briefly described their interrelation. This section focuses on the evaluation of CONVERGENCE with the help of the two applications.

\subsection{Study Design}

We conducted a user study over a period of one week, where students and lecturers had to complete several tasks with the podcast creator application and the augmented lecture podcast service. After this, participants were invited to a focus group, during which they were introduced to the main ideas of the CONVERGENCE framework and where they had the possibility to ask questions. Altogether, 11 Lecturers and 13 students took part in the user study and focus groups. All lecturers were research assistants; all students had a background in computer science.

\subsection{Study Tasks}

Since lecturers and students tested different applications, they were assigned different tasks. While lecturers had to accomplish task related to the podcast creation process, students had to complete task within a social learning environment.

Lecturers were instructed to repeat the same tasks three times within the week of the user study. The first task was to register for the podcast creator application (obviously, this only had to be done once) and login to the application where they were asked to create and publish a podcast (this involved the description of podcasts with metadata, the setting of an expiration date, etc.). Once this task was completed, they had to update some podcast information and finished with the deletion of the updated podcast.

Students had to perform similar tasks that they had to repeat three times within the week of the user study. The first task was to register for the augmented lecture podcast service (only once) and login to the application, where they had to search for a certain podcast, view the results and choose the corresponding podcast for watching. Furthermore, students were asked to annotate the podcast they were watching and to subscribe to at least on annotation written and published by another student.

\subsection{Results}

In general, students and lecturers had similar perceptions about the CONVERGENCE framework. For all of them, it was quite difficult to grasp all functionalities provided by CONVERGENCE, since they were hidden underneath the application and not immediately visible on the actual user interface (e.g. the creation and publication of VDIs is transparent to the user and accomplished in the background of the application).

"I did not notice that there is a different system beneath the application” (quote by participant)

"The technical background of the application is not
evident." (quote by participant)

Nonetheless, participants found the goals of CONVERGENCE appealing. In particular, the synchronization of content was one of the most attractive aspects for our participants. They liked the idea to work with content that is always up to date. The participants also saw the opportunity to have better control over their own published content (e.g by defining licenses and setting an expiration date). However, participants are still skeptical and are afraid, that these new features might come with some overhead and disadvantages when creating content.

\section{"The old Internet is simple and we know its problems. The biggest problem with \\ CONVERGENCE is that it poses new kinds of problems that are yet unknown." (quote by participant)}

For example, one user hinted that by using an expiration date, the Internet will lose important information. Since one content might be unimportant for one person, but may be valuable for another person.

In summary, participants are intrigued by the concepts of CONVERGENCE (expiration date, licensing, digital forgetting, etc.) within a podcasting application, but they have a rather vague picture of what CONVERGENCE actually is, since most functionality is hidden underneath the application. However, this is not necessarily a big disadvantage for the adoption of CONVERGENCE, since it is an underlying technology.

"To me it does not matter on which system the application runs, most importantly it is functional." (quote by participant)

\section{Conclusion}

In this paper, we gave an overview of state of the art podcasting solutions, introduced the concept of an information centric network and exemplarily showed how podcasting applications can be employed using the CONVERGENCE information-centric network. 
Only a few lines of code are sufficient to implement a powerful functionality that is based on publishsubscribe mechanisms and that can be easily used to connect two applications with each other in order to share content.

Our study revealed that the functionalities provided by CONVERGENCE are transparent to the user. This should not be considered negatively, since CONVERGENCE does not change the way we use certain functionality within an application, but moreover, it changes the way certain functionality is achieved.

In future work the presented applications are supposed to be refined and enriched with additional CONVERGENCE features. The goal is to develop a fully functional social learning environment and connect it with other educational applications to employ the actual advantages of CONVERGENCE. Once this is done, another user study will be conducted over a longer period of time, so that users will be able to perceive the advantages that CONVERGENCE provides (content that expires, notifications when new content is published).

Similar to the evolution of the current Internet, information centric networks need their time to unfold, since only over time, users will be able to see the power of this new concept.

\section{Acknowledgements}

The authors would like to thank all the CONVERGENCE partners for the fruitful collaboration, in particular those who have contributed to the development of the CONVERGENCE use cases.

\section{References}

[1] T. O' Reilly, "What Is Web 2.0 Design Patterns and Business Models for the Next Generation of Software", O' Reilly Network, 2005, http://facweb.cti.depaul.edu/jnowotarski/se425/What\%20Is \%20Web\%202\%20point\%200.pdf

[2] S. Banister, "Web 2.0 tools in the reading classroom: teaching exploring literacy in the $21^{\text {st }}$ century", International Journal of Technology in Teaching and Learning, 4(2), pp. 109-116.

[3] M. Chui, A. Miller, and R. Roberts, "Six ways to make Web 2.0 work”, McKinsey Quarterly, 1, 2009, pp. 2-7.

[4] N. C. Linh, "A survey of the application of Web 2.0 in Australasian universities libraries”, Lib. Hi. Technology, 26(4), 2008, pp. 630-653.
[5] A. Rosen, “Technology trends: e-learning 2.0", The e-learning guild's learning solutions e-magazine, 2006, http://www.readygo.com/e-learning-2.0.pdf

[6] G. Cormode and B. Krishnamurthy, "Key differences between Web 1.0 and Web 2.0”, AT\&T Labs-Research White Paper, 2008, http://www2.research.att.com/ bala/papers/web1v2. pdf

[7] B. Roxin, and I. Smeureanu, "E-learning platforms for semantic web", in Proceedings of the 20th World Conference on Educational Multimedia Hypermedia \& Telecommunications (EDMEDIA), Vienna, Austria, 2008, pp. 1695-1699

[8] F. Pedró, "The new millennium learners: challenging our views on ICT and learning”, OECDCERI, http://www.oecd.org/dataoecd/1/1/38358359.pdf

[9] D. Baird, and M. Fisher, "Neomillennial user experience design strategies: utilizing social networking media to support 'always on' learning styles”, Journal of Educational Technology Systems, 34, 2006, pp. 5-32.

[10] G. Siemens, "Knowing Knowledge”, 2006, http://www.knowingknowledge.com

[11] A. Bruns, and S. Humpheys, "Building collaborative capacities in learners: the M/encyclopedia project revisited", in Proceedings of the International Symposium on Wikis, Montreal, Canada, 2007, pp. 1-10

[12] C. Redecker, K. Ala-Mutka, M. Bacigalupo, A. Ferrari, and Y. Punie, "Learning 2.0: the impact of web2.0 innovations on education and training in Europe”, JRC Scientific and Technical Report, 2009

[13] P. Meng, "Podcasting \& Vodcasting: Definitions, Discussion \& Implications”, IAT Services at University of Missouri, 2005, http://edmarketing.apple.com/adcinstitute/wp_conten t/Missouri_Podcasting_White_paper.pdf

[14] A. Gronstedt, "Basics of Podcasting: Tips, Tools and Intelligence for Trainers”, Infoline White Papers, 2007, http://www.gronstedtgroup.com/site_2011/pdf/Infoli ne_podcasting.pdf

[15] M. Lazzari, and A. Betella, Towards Guidelines on Educational Podcasting Quality, In M. Smith and G. Salvendy (Eds.) Human Interface and the Management of Information, Lecture Notes in Computer Science, Springer-Verlag, 2007 
[16] G. Tyson, A Middleware Approach to Building Content-Centric Applications, PhD thesis, Lancaster University, 2010

[17] D. Smetters, and V. Jacobson, "Securing network content”, PARC TR White Papers, 2009, pp. 1-7

[18] D. Perino, and M. Varvello, “A reality check for content centric networking”, in Proceeding of ACM SIGCOMM Workshop, Toronto, Canada, 2011, pp. 44-49

[19] N. Melazzi, “Convergence: Extending the Media Concept to Include Representations of Real World Objects", in Proceedings of the 20th Tyrrhenian International Workshop on Digital Communications, Pula, Italy, 2009. pp. 129-140

[20] I. Burnett, S. Davis, G. Drury, "MPEG-21 Digital Item Declaration and Identification Principles and Compression”, IEEE Transactions on Multimedia, 7(3), 2005, pp. 400-407 